\title{
Oral proteasome inhibitor with strong preclinical efficacy in myeloma models
}

Jonghoon Park', Eok Park', Cheol-Kyu Jung', Seung-Wan Kang, Byung Gyu Kim¹, Youngjoo Jung ', Tae Hun Kim', Ji-Young Lim², Sung-Eun Lee², Chang-Ki Min² and Kwang-Ai Won ${ }^{1 *}$

\begin{abstract}
Background: The proteasome is a validated anti-cancer target and various small-molecule inhibitors are currently in clinical development or on the market. However, adverse events and resistance associated with those proteasome inhibitors indicate the need for a new generation of drugs. Therefore, we focused on developing an oral proteasome inhibitor with improved efficacy and safety profiles.

Method: The in vitro inhibition of the 20S proteasome catalytic activities was determined in human multiple myeloma (MM) cellular lysates with fluorogenic peptide substrates specific for each catalytic subunit. Cell cytotoxicity was assessed with the ATP bioluminescence assay using human cell samples from tumor cell lines, MM patients or normal healthy donors. In mice bearing human MM xenografts, a single dose of LC53-0110 was administered orally, and concentrationtime profiles of LC53-0110 and the 20S proteasome catalytic activities in plasma, blood, and tumor were determined. The efficacy of repeat-dose compound with regard to tumor growth inhibition in vivo was also evaluated in the same MM xenograft models.
\end{abstract}

Results: LC53-0110 is far more specific for the chymotrypsin-like proteolytic ( $\beta 5$ ) site of the 20 s proteasome as compared to bortezomib, carfilzomib, or ixazomib. LC53-0110 treatment showed accumulation of ubiquitinated proteins, inhibited cell viability with a low nM range potency in various tumor cell lines, and showed potent activity on CD138 ${ }^{+}$cells isolated from MM patients who are resistant/refractory to current FDA-approved drug treatment. When a single dose was administered orally to tumor-bearing mice, LC53-0110 showed both greater maximum and sustained tumor proteasome inhibition as compared with ixazomib in MM xenograft models. The robust pharmacodynamic responses in tumor correlated with tumor growth regression. In addition, LC53-0151, an analog of LC53-0110, in combination with pomalidomide, a third-generation immunomodulatory drug, showed synergistic inhibition of tumor growth both in vitro and in the xenograft mouse model.

Conclusions: In view of the in vitro, in vivo, and ex vivo profiles, further investigation of additional LC compounds in preclinical studies is warranted for the nomination of a clinical development candidate.

Keywords: Multiple myeloma, Proteasome inhibitor, Oral drug, Combination therapy, Pomalidomide

\section{Background}

The $26 \mathrm{~S}$ proteasome is a multimeric complex consisting of a centrally-located $20 \mathrm{~S}$ core catalytic complex flanked by 19S regulatory subunits [1-3]. The 19S caps contain multiple ATPase active sites and ubiquitin binding sites which recognize and unfold ubiquitinated protein substrates and transfer them to the 20S core. The 20S complex contains two outer $\alpha$ rings and two inner $\beta$ rings. Each $\beta$ ring

\footnotetext{
* Correspondence: wonk12pharm@yahoo.com

${ }^{1}$ R\&D Center, LG Life Sciences, Ltd, Daejeon, South Korea

Full list of author information is available at the end of the article
}

contains three active enzymatic sites with distinct substrate specificities: chymotrypsin-like (CT-L) on the $\beta 5$ subunit, trypsin-like (T-L) on the $\beta 2$ subunit, and caspase-like (C-L) on the $\beta 1$ subunit. The proteasome plays a critical role in cellular homeostasis by degrading a variety of proteins involved in cell cycle control, signal transduction, apoptosis, antigen processing, cell differentiation and proliferation through the ubiquitin-proteasome pathway. Therefore, the sites of the 26S proteasome, both 19S and 20S subunits, have been drug targets for various disease indications including cancer [4]. 
Bortezomib, a drug administered intravenously or subcutaneously, was the first proteasome inhibitor targeting enzymatic sites of the $\beta$ chain and validated the proteasome as an anti-cancer target $[5,6]$. It was approved for the treatment of mantle cell lymphoma and MM. Since FDA-approval of bortezomib, second-generation proteasome inhibitors with improved therapeutic indices have been pursued in various systems ranging from virtual modeling to tumor-bearing animals [7, 8]. Recently, carfilzomib also administered intravenously, was approved for the treatment of refractory MM [9-11]. There are currently other various small-molecule inhibitors in clinical development including ixazomib [12], oprozomib [13], and marizomib [14]. Whereas ixazomib and oprozomib are orally bioavailable proteasome inhibitors, marizomib is an intravenously administered inhibitor with less protease specificity.

Here, we describe the characterization of the preclinical pharmacology of LC53-0110 and its analog which are novel, reversible, selective, potent, and orally bioavailable proteasome inhibitors. We investigated the effects of LC53-0110 on proteasome CT-L activities in vitro and in tumor cell lines, and addressed the anti-cancer mechanism by analyzing apoptosis pathways. Furthermore, we validated its effects on primary human myeloma cells of multiple myeloma patients. Finally, the compound's in vivo effects were evaluated in multiple myeloma xenograft mouse models.

\section{Methods}

\section{Human tumor cell lines and chemicals}

Cell lines were obtained from the American Type Culture Collection (ATCC) and maintained in culture conditions at $37{ }^{\circ} \mathrm{C}$ under $5 \% \mathrm{CO}_{2}$. RPMI8226, MM.1S, U2932, SuDHL-8, H1650, NCI-H1975, MDA-MB-231, and MCF-7 cells were maintained in RPMI-1640 (Gibco) containing $10 \%$ fetal bovine serum (FBS, heat-inactivated; Gibco) and $1 \%$ penicillin-streptomycin-amphotericin B (Gibco). HCT116 and HT-29 cells were maintained in McCoy's 5A medium (Gibco) containing $10 \%$ FBS and $1 \%$ penicillinstreptomycin-amphotericin B.

Bortezomib, ixazomib, and carfilzomib were synthesized in our laboratory and the structures were confirmed by nuclear magnetic resonance. Pomalidomide was purchased from Tokyo Chemical Industry. Fluorogenic peptide substrates Suc-Leu-Leu-Val-Tyr-AMC (7-amido-4-methyl-coumarin) for the proteasomal CT-L activity, ZAla-Arg-Arg-AMC for the proteasomal T-L activity, and Z-Leu-Leu-Glu-AMC for the proteasomal C-L activity were purchased from Calbiochem, EMD Millipore.

\section{In vitro enzyme assays}

RPMI8226 cell lysate was used for the in vitro proteasome inhibition assay. Cells were washed with PBS and resuspended in $20 \mathrm{mM}$ Tris- $\mathrm{HCl}, \mathrm{pH}$ 7.5. Then, cells were lysed on ice by dounce homogenization and the supernatants were collected by centrifugation. The assay was conducted in 96-well plates by incubating $2 \mu \mathrm{g}$ of lysates with serial dilutions of a test compound and $20 \mu \mathrm{M}$ of substrate at $37{ }^{\circ} \mathrm{C}$ for $1 \mathrm{~h}$. After incubation, production of hydrolyzed AMC groups was measured using a FlexStation II Fluorometer (Molecular Devices) or using a Spectra MAX Gemini Fluorometer (Molecular Devices) with an excitation filter of $380 \mathrm{~nm}$ and an emission filter of $460 \mathrm{~nm}$.

The non-proteasome protease panel assay was conducted at $10 \mu \mathrm{M}$ of a test compound using standard substrate-based assays (GenScript, NJ). After $10 \mathrm{~min}$ incubation of proteases and inhibitor compounds at room temperature, substrates for respective proteases were added to initiate the reactions, and products from each reaction were analyzed with PHERAstar Plus (BMG LABTECH) or FlexStation 3 (Molecular Devices) using kinetics or endpoint model. Percent inhibition was calculated as [1- (sample activity-substrate control)/(enzyme activity-substrate control)] $\times 100$. The positive control of each non-proteasome protease showed 96-103 \% inhibition, thereby validating the assay performance.

\section{Cell viability assays}

Cell cytotoxicity was assessed using the ATP Bioluminescence Assay. RPMI8226, MM.1S, U2932, Su-DHL-8 cells were plated onto 96-well microtiter plates in culture medium and then treated with a serial dilution of compound in medium containing a final concentration of $0.5 \%$ DMSO. Cultures were incubated for $48 \mathrm{~h}$ and cells were then assayed for viability using the CellTiterGlo Luminescent Cell Viability Assay kit (Promega). For the adherent cell lines, cells were plated and incubated for $24 \mathrm{~h}$. Cells were then treated with a serial dilution of compound for an additional $72 \mathrm{~h}$ and assayed for viability.

For the MTT [3-(4,5-dimethythiazol-2-yl)-2,5-diphenyltetrazolium bromide] assay, MM.1S cells were plated and incubated with DMSO or varying concentrations of LC53-0151 and pomalidomide. At the end of the incubation, the water soluble MTT was added and cell viability was determined by measuring optical density at $560 \mathrm{~nm}$ using SpectraMax M5e (Molecular Devices).

\section{Western immunoblot analysis}

RPMI8226 cells were seeded and treated with either 500 $\mathrm{nM}$ of test compounds or $0.1 \%$ DMSO. After incubation for $1 \mathrm{~h}$, cells were washed with media three times, and incubated for an additional 4 or $24 \mathrm{~h}$. Cells were washed with ice-cold PBS and lysed with Pro-Prep lysis buffer (iNtRON Biotechnology). Protein lysates were analyzed by Western immunoblotting using the following antibodies: anti-Ubiquitin (Enzo Life Sciences), anti-Hsp70 
and anti-Hsp27 (Abcam), anti-Gadd34 (Proteintech), anticaspase-8 (BD pharmingen), anti- $\beta$-Catenin, anti-p21, anti-phospho Hsp27 (S82), anti-caspase-9, anti-PARP, and anti- $\alpha$-Tubulin (Cell Signaling). Images were obtained using ChemiDoc MP (Bio-Rad) and quantitation was done using Image Lab Software (Bio-Rad).

\section{Human primary cell samples}

All human cell samples from MM patients and normal healthy donors were obtained after approval by the Institutional Review Board (IRB) for Human Research at the Catholic University of Korea and independent Ethics Review Board at the LG Life Sciences with written informed consent, respectively.

Primary MM cells were retrieved from bone marrow of patients and were enriched by Ficoll density centrifugation to a purity of $>90 \%$, as necessary. Purity was assessed by cytomorphology on stained samples. CD138 ${ }^{+}$cells were collected using magnetic beads (Miltenyi Biotec) and plated at a density of $8 \times 10^{4}$ cells per well onto 96-well plates in RPMI-1640 (WELGENE) containing 10 \% FBS (WELGENE), $1 \%$ penicillin-streptomycin-amphotericin B (Gibco), and $2 \mathrm{mM} \mathrm{L-glutamine.} \mathrm{Cells} \mathrm{were} \mathrm{then} \mathrm{treated}$ with a serial dilution of compound in medium containing a final concentration of $0.2 \%$ DMSO. Cultures were incubated for $48 \mathrm{~h}$ and cells were then assayed for viability using the CellTiter-Glo Luminescent Cell Viability Assay kit (Promega).

Peripheral blood mononuclear cells (PBMCs) isolated from the heparinized blood samples of normal healthy donors were also plated and processed as described above.

\section{Tumor-bearing mouse studies}

In vivo efficacy studies were performed in female BALB/ c nude mice (8 weeks old) purchased from ORIENT Bio or female NOD.SCID mice (11-13 weeks old) purchased from HFK Bioscience. The animals were housed and maintained in a controlled environment including a $12 \mathrm{~h}$ light and $12 \mathrm{~h}$ dark cycle with automatic timers and received food and water ad libitum. All experiments were done under protocols approved by the Institutional Animal Care and Use Committee (IACUC). Moribund animals were sacrificed when necessary and animal carcasses were disposed of according to IACUC guidelines.

$1 \times 10^{7}$ MM.1S tumor cells in $0.1 \mathrm{ml}$ of Dulbecco's Phosphate-Buffered Saline (1:1 matrigel) were implanted subcutaneously into the right flank of female athymic nude mice. Tumor growth was monitored with calipers and mice were divided into homogeneous cohorts according to their initial tumor volume. Then within each cohort, mice were randomized to treatment groups just before dose initiation, thus ensuring that all the groups have comparable baselines. The compounds were formulated in $0.5 \%$ methyl cellulose and administered at the indicated doses and regimens by oral gavage at a dose volume of $10 \mathrm{~mL} / \mathrm{kg}$. For the RPMI8226 model, a total of $1 \times$ $10^{7}$ cells in $0.2 \mathrm{ml}$ of PBS (1:1 matrigel) were implanted subcutaneously into the hind flank of female NOD.SCID mice. Whole blood was collected by cardiac puncture into tubes containing sodium heparin and cells were harvested by centrifugation. Blood cell pellets and tumor tissues were homogenized in lysis buffer $(50 \mathrm{mM}$ Tris- $\mathrm{HCl}$, pH 8.0, 5 mM EDTA, $150 \mathrm{mM} \mathrm{NaCl}, 0.5 \%$ Nonidet P-40, $0.5 \mathrm{mM}$ phenylmethylsulfonyl fluoride, and $0.5 \mathrm{mM}$ dithiothreitol) for $30 \mathrm{~min}$ at $4{ }^{\circ} \mathrm{C}$. The lysates were centrifuged at $14,000 \times \mathrm{g}$ for $30 \mathrm{~min}$, and the supernatants were collected as whole blood cell extracts and tumor cell extracts, respectively, for pharmacodynamic analysis. Blood, plasma, and tumor tissue samples were extracted with acetonitrile $(0.1 \%$ formic acid) containing internal standard, followed by centrifugation at $3600 \mathrm{rpm}$ for $10 \mathrm{~min}$. Supernatant was injected into the LC-MS/MS system for pharmacokinetic analysis. The mean, standard deviation (SD), and standard error of the mean (SEM) were calculated for each experimental group, and statistical analyses were performed using standard two tailed Student's $t$-test (significance defined as $P \leq 0.05$ ).

\section{Results}

\section{LC53-0110 is a selective inhibitor of CT-L activity}

LC53-0110 was identified following optimization of a new scaffold identified in silico for in vitro inhibition of proteasome CT-L activities using the fluorogenic substrate succinyl-Leu-Leu-Val-Tyr-AMC and human MM RPMI8226 cell lysates. LC53-0110 displayed potent inhibitory activity against $\mathrm{CT}$-L, with $\mathrm{IC}_{50}$ values in the single $\mathrm{nM}$ range (Table 1). LC53-0110 was less potent against the other proteolytic sites of the $20 \mathrm{~S}$ proteasome, i.e., $\mathrm{T}$ - $\mathrm{L}$ and $\mathrm{C}$ - $\mathrm{L}$, with $\mathrm{IC}_{50}$ values of $>20,000 \mathrm{nM}$ and 600-1300 $\mathrm{nM}$, respectively. When the relative potency of LC53-0110 was compared side by side with other proteasome inhibitors, LC53-0110 was found to be more specific toward CT-L inhibitory activity. LC53-0110 was

Table 1 Inhibition of the proteasome

\begin{tabular}{lllll}
\hline \multirow{2}{*}{$\begin{array}{l}\text { Compound (route } \\
\text { of administration) }\end{array}$} & \multicolumn{2}{l}{ Proteasome inhibition, $\mathrm{IC}_{50}(\mathrm{nM})$} & \multirow{2}{*}{$\begin{array}{l}\text { Binding } \\
\text { kinetics }\end{array}$} \\
\cline { 2 - 4 } & $\mathrm{CT}-\mathrm{L}, \beta 5$ & $\mathrm{~T}-\mathrm{L}, \beta 2$ & $\mathrm{C}-\mathrm{L}, \beta 1$ & \\
\hline Bortezomib (IV/SC) & $2 \sim 4$ & $2400 \sim 3700$ & $16 \sim 29$ & reversible \\
Carfilzomib (IV) & $7 \sim 9$ & $400 \sim 460$ & $420 \sim 590$ & irreversible \\
Ixazomib (PO) & $3 \sim 5$ & $>10000$ & $6 \sim 7$ & reversible \\
LC53-0110 (PO) & $1 \sim 5$ & $>20000$ & $600 \sim 1300$ & reversible \\
\hline
\end{tabular}

The in vitro inhibition of the $20 \mathrm{~S}$ proteasome catalytic activities was determined in RPMI8226 cellular lysates with fluorogenic peptide substrates specific for each catalytic subunit. Three reference compounds were tested in the assays along with LC53-0110. Binding kinetics was determined by assessing CT-L activity in RPMI8226 cells at different time points after 1-h pulse treatment of cells with a compound.

CT-L chymotrypsin-like, T-L trypsin-like, C-L caspase-like. IV intravenous, SC subcutaneous, $P O$ per os, by mouth 
determined to be a reversible inhibitor when CT-L activity was assessed in vitro at different time-points after RPMI8226 cells were pulse-treated for $1 \mathrm{~h}$ with the compound (data not shown). When assessed against representative non-proteasomal proteases (Fig. 1), LC53-0110 had relatively weak or no inhibitory activities except against neutrophil Elastase (serine protease with P1 specificity of valine), for which it showed $65 \%$ inhibition at $10 \mu \mathrm{M}$, a potency similar to that of bortezomib and ixazomib. It is noteworthy that LC53-0110 differentiated itself from those proteasome inhibitors by showing no inhibitory activity against Cathepsin G (serine protease with P1 specificity of phenylalanine/leucine/tyrosine).

\section{LC53-0110 inhibits viability in human tumor cell lines}

When effects on cell viability were determined by bioluminescent measurement of cellular ATP, LC53-0110 inhibited viability in RPMI8226 cells with $\mathrm{IC}_{50}$ values of 6-12 $\mathrm{nM}$ at $48 \mathrm{~h}$ (Table 2). In additional tumor cell lines with diverse origins and genetic backgrounds, LC530110 was found to inhibit viability with $\mathrm{IC}_{50}$ values ranging from $3 \mathrm{nM}$ to $25 \mathrm{nM}$, demonstrating potency in both hematologic and solid tumor cell lines tested.

For the assessment of the compound's effect on the proteasome-dependent protein degradation pathway, cell lysates were prepared from RPMI8226 four hours after a one-hour pulse treatment with a compound and assessed by Western blot analysis with an ubiquitinspecific monoclonal antibody. The level of ubiquitinated proteins with a group of slower migrating bands in cells treated with LC53-0110 or its analog LC53-0151 were increased as compared to DMSO-treated cells (Fig. 2a), confirming inhibition of proteasome protease



Fig. 1 Inhibition of non-proteasomal proteases. $10 \mu \mathrm{M}$ of proteasome inhibitor compounds were tested with a panel of proteases, Cathepsin $\mathrm{G}$ (serine protease with P1 specificity of phenylalanine/leucine/tyrosine), Elastase (neutrophil serine protease with P1 specificity of valine), Cathepsin B (cysteine protease), Renin (aspartate protease), and ACE (metalloprotease angiotensin converting enzyme). Percent inhibition was calculated based on compound's effect on protease activity subtracted with a substrate control without an enzyme
Table 2 Inhibition of cellular viability

\begin{tabular}{lll}
\hline Cell line & Tumor type & $\mathrm{IC}_{50}(\mathrm{nM})$ \\
\hline RPMI8226 & Multiple myeloma & $6 \sim 12$ \\
MM.1S & Multiple myeloma & $3 \sim 6$ \\
U2932 & Activated B-cell like diffuse large B-cell lymphoma & $6 \sim 9$ \\
SU-DHL-8 & Diffuse large B-cell lymphoma & $11 \sim 18$ \\
HCT116 & Colorectal carcinoma & $13 \sim 14$ \\
HT-29 & Colorectal adenocarcinoma & $11 \sim 16$ \\
H1650 & Lung carcinoma & $7 \sim 22$ \\
NCI-H1975 & Lung carcinoma & $2 \sim 14$ \\
MDA-MB-231 & Breast adenocarcinoma & $8 \sim 14$ \\
MCF-7 & Breast adenocarcinoma & $14 \sim 25$
\end{tabular}

The cytotoxic effects of LC53-0110 on a panel of tumor cell lines were determined by bioluminescent measurement of cellular ATP using CellTiter-Glo reagent.

Hematologic and solid tumor cells were incubated with the compound for $48 \mathrm{~h}$ or $72 \mathrm{~h}$, respectively prior to assay

activities in cells, thereby blocking the proteasomedependent polyubiquitinated protein degradation pathway. The inhibition of proteasome function was also confirmed by accumulation of the direct proteasome substrates p21 and $\beta$-Catenin (Fig. 2b).

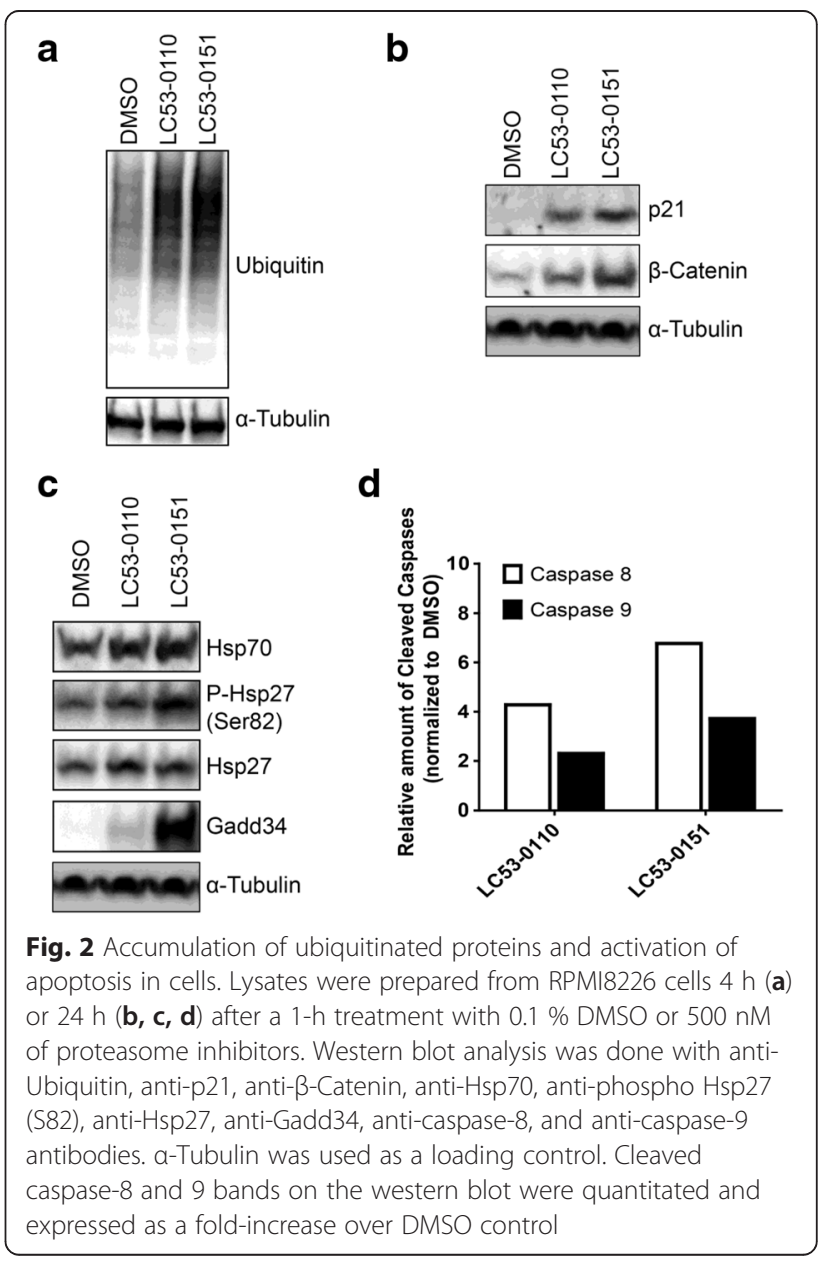


Since accumulation of excessive or misfolded proteins triggers the cellular stress response including upregulation of heat shock protein (Hsp) expression and the unfolded protein response (UPR) [15-17], some of these components were examined to assess the mechanism of action of LC53-0110 and its analog LC53-0151. In RPMI8226 cells, significant basal levels of Hsp70, Hsp27, and phospho-Hsp27 were detected (Fig. 2c). Upon treatment with either compound, levels of Hsp70 and Hsp27 were increased, suggesting a collective cellular protection response by molecular chaperones. Phosphorylation of Ser82, one of the main phosphorylation sites of Hsp27 in vivo by MAP kinase-activated protein kinase 2 (MAPKAPK2) [18], was also increased, likely affecting its chaperone activity [19]. GADD34 was greatly induced upon compound treatment, suggesting UPRdependent ER stress-induced cell death [15]. Therefore, to further address the mechanism of compound effects on cell viability, two types of caspases were assessed for activation of the apoptotic cell death pathway (Fig. 2d, Additional file 1: Figure S1A). Whereas caspase-8 triggers the apoptotic signal transduction cascade upon binding of death receptor ligands to their corresponding receptors, caspase-9 links induction of stress signaling pathways to the mitochondrial death pathway [20,21]. Since activation of these caspases is associated with the cleavage of their inactive pro-enzyme forms, the level of cleaved caspases were determined in RPMI8226 cells. LC53-0110 and its analog LC53-0151 increased cleaved caspase- 8 levels four to seven-fold and caspase- 9 levels two to four-fold, respectively. Therefore, both compounds triggered mitochondria-dependent and -independent apoptotic cell death signaling pathways, thus leading to cleavage of the apoptotic marker poly(ADP-ribose) polymerase (PARP) (Additional file 1: Figure S1B). Treatment of human multiple myeloma MM.1S cells with either LC53-0110 or LC53-0151 also showed similar results (data not shown).

\section{LC53-0110 inhibits viability in primary human myeloma cells}

To assess effects of LC53-0110 on viability of primary human myeloma cells, CD $138^{+}$cells were purified from bone marrow samples of MM patients who had been either newly diagnosed or exposed to various standard therapies (Table 3). When effects on cell viability were determined by bioluminescent measurement of cellular ATP, the median $\mathrm{IC}_{50}$ for LC53-0110 and a range value were $15 \mathrm{nM}$ and $32 \mathrm{nM}$, respectively (Fig. 3b, Additional file 2: Figure S2). In patient 4 and patient 14 samples where $\mathrm{IC}_{50} \mathrm{~s}$ could not be determined due to ambiguous slopes, viability at $12.5 \mathrm{nM}$ was 15 and $10 \%$, respectively (Fig. 3a). Therefore, regardless of prior therapy regimens applied to the
Table 3 Summary of multiple myeloma patients

\begin{tabular}{|c|c|c|c|c|c|c|c|}
\hline Patient & Age & Type & D-S stage & $V^{R}$ & $T^{R}$ & $L^{R}$ & $\begin{array}{l}\text { Number of } \\
\text { treatment lines }\end{array}$ \\
\hline 11 & $>65$ & $\lg G-\lambda$ & IIla & N.A. & N.A. & N.A. & naive \\
\hline 4 & $>65$ & $\lg G-K$ & Illa & N.A. & N.A. & N.A. & naive \\
\hline 8 & $<65$ & $\lambda$ & Illa & Y & N.A. & Y & 4 \\
\hline 15 & $>65$ & $\lg A-\lambda$ & Illa & Y & N.A. & Y & 2 \\
\hline 3 & $<65$ & $\lg G-\lambda$ & IIla & Y & Y & Y & 4 \\
\hline 16 & $>65$ & $\lg G-K$ & Illa & Y & Y & Y & 6 \\
\hline 14 & $<65$ & $\lambda$ & $\| l \mathrm{~b}$ & Y & Y & Y & 3 \\
\hline 6 & $<65$ & $\lg G-K$ & Illa & Y & $\mathrm{N}$ & N.A. & 2 \\
\hline 7 & $<65$ & $\lambda$ & $\mathrm{lllb}$ & $\mathrm{N}$ & Y & $Y$ & 3 \\
\hline
\end{tabular}

D-S Stage: The Durie-Salmon staging system. V: bortezomib. T: thalidomide. L: lenalidomide. R: resistance or refractory. N.A.: Not Applicable. Type: paraproteins $\lg G$ or $\lg A$, $k$-light chains $\mathrm{k}$ or $\lambda$-light chains $\lambda$

a For patients who received autologous stem cell transplantation (ASCT), a regimen of induction + ASCT+/-maintanence was counted as one treatment line. Naive: no past treatment

patients and whether or not the patients became resistant or refractory, LC53-0110 showed a potent inhibition of viability in primary myeloma cells from the patients. The reference compound ixazomib was less potent than LC53-0110 in those patient samples (Fig. 3a). When normal PBMC samples from healthy donors were assessed for cytotoxicity, LC53-0110 and ixazomib showed more than $50 \%$ viability at 10,000 $\mathrm{nM}$, the highest concentration tested (data not shown). Therefore, normal PBMC cell viability was relatively less affected by proteasome inhibitors as compared with that of myeloma cells.

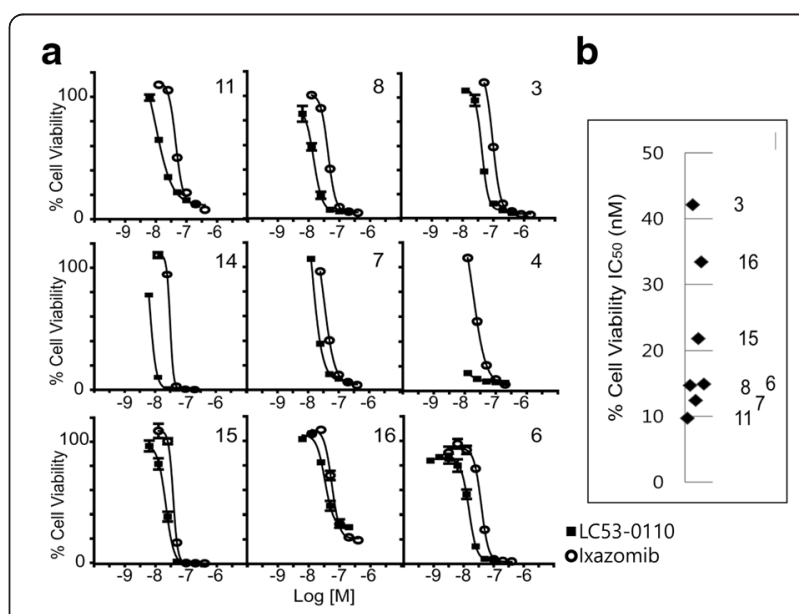

Fig. 3 Inhibition of cellular viability in patient-derived samples. a CD138 cells were purified from the bone marrow samples of MM patients $(3,4$, 6-8, 11, 14-16). The cytotoxic effects of LC53-0110 (filled rectangle) and ixazomib (open circle) were determined by bioluminescent measurement of cellular ATP using CellTiter-Glo reagent after $48 \mathrm{~h}$ of treatment. Results are expressed as \% cell viability over DMSO control. b The IC50 values for LC53-0110 treated MM patients 


\section{LC53-0110 strongly inhibits proteasome catalytic activity in tumor xenograft models}

Having established anti-viability in tumor cell lines, systemic effects of LC53-0110 on tumor growth in an organism were assessed. MM.1S xenograft tumors were subcutaneously implanted in the hind flank of athymic nude mice and the ability of LC53-0110 to inhibit endogenous proteasomal CT-L activity was examined following a single oral dose of $50 \mathrm{mg} / \mathrm{kg}$. The tumors were harvested $1,7,24,48$ or $72 \mathrm{~h}$ after treatment and homogenized in lysis buffer. Blood was collected at the same time as tumor tissues were harvested, and blood lysates were prepared as well. The inhibition of proteasomal CT-L catalytic activity in the tumors was $82 \%$ at $1 \mathrm{~h}$, reached a maximum of $97 \%$ at $48 \mathrm{~h}$, and persisted through $72 \mathrm{~h}$ (Fig. 4a). In contrast, the inhibition of CT-L catalytic activity in the blood decreased from $72 \%$ at $1 \mathrm{~h}$ to $29 \%$ at $7 \mathrm{~h}$, and further decreased with only minimal or no inhibition evident by $72 \mathrm{~h}$.

The concentrations of LC53-0110 in plasma, blood, and tumors were also determined in this study (Fig. 4b). Tumor concentrations were $1 \mu \mathrm{M}$ at $1 \mathrm{~h}$ and decreased to $0.4 \mu \mathrm{M}$ at $7 \mathrm{~h}$ and $24 \mathrm{~h}$. Then, the compound levels gradually decreased to $0.1 \mu \mathrm{M}$ by $72 \mathrm{~h}$. In contrast, blood concentrations were $1.8 \mu \mathrm{M}$ at $1 \mathrm{~h}$ and continuously decreased to $0.03 \mu \mathrm{M}$ by $72 \mathrm{~h}$. The slope of concentration change in plasma over time was similar to that in blood with $3.3 \mu \mathrm{M}$ at $1 \mathrm{~h}$ and $0.04 \mu \mathrm{M}$ by $72 \mathrm{~h}$. Therefore, whereas $97 \%$ inhibition of tumor CT-L activity occurred at the tumor concentration of $0.2 \mu \mathrm{M}, 14 \%$ inhibition of blood CT-L activity occurred at the blood concentration of $0.16 \mu \mathrm{M}$. The data presented here show the pharmacodynamic effect of LC53-0110 to be greater in tumors over blood as well to outlast a detectable plasma or blood exposure.

\section{LC53-0110 displays robust anti-tumor activity in tumor xenograft models}

The efficacy of repeat-dose LC53-0110 with regard to tumor growth inhibition in vivo was evaluated in the previously described MM.1S as well as RPMI8226 xenograft models. Due to its durable target modulation activity, i.e., a sustained pattern of proteasomal CT-L inhibition in tumors, it could be predicted that dosing every several days or even once a week with LC53-0110 might result in significant anti-tumor efficacy in vivo. Indeed, when administered orally at $50 \mathrm{mg} / \mathrm{kg}$ of LC53-0110 (Fig. 5a), MM.1S tumor regression was observed on day 4 postdosing (42-52\% of start tumor volume) and almost complete tumor regression was achieved by day 15 with all dosing regimens tested (twice or once a week schedules). In contrast, when orally administered twice a week at $5 \mathrm{mg} / \mathrm{kg}$, ixazomib showed a maximum tumor regression on day 8 (36\% of start tumor volume), after which the tumors appeared to regrow, reaching $58 \%$ of start tumor volume and $72 \%$ tumor growth inhibition on day 15 as compared to the vehicle control group.

The strong efficacy of LC53-0110 was also evident in the RPMI8226 xenograft model. Dose-dependent tumor growth inhibition was achieved by LC53-0110 at $25 \mathrm{mg} /$ $\mathrm{kg}(56 \%)$ and $50 \mathrm{mg} / \mathrm{kg}(82 \%)$ on day 22 when orally administered twice a week (Fig. 5b). Tumor growth inhibition by LC53-0110 dosed $50 \mathrm{mg} / \mathrm{kg}$ once a week was similar to that seen with the twice a week dosing regimen throughout the treatment period. Moreover, tumor growth inhibition by $50 \mathrm{mg} / \mathrm{kg}$ LC53-0110 administered orally was greater than $0.6 \mathrm{mg} / \mathrm{kg}$ bortezomib or $2.0 \mathrm{mg} / \mathrm{kg}$ carfilzomib, both of which were administered intravenously. The anti-tumor effect of LC53-0110 as assayed by tumor volume change was also confirmed by weight measurement of tumors extracted after final tumor volume measurement in tumor-bearing mice (data not shown).
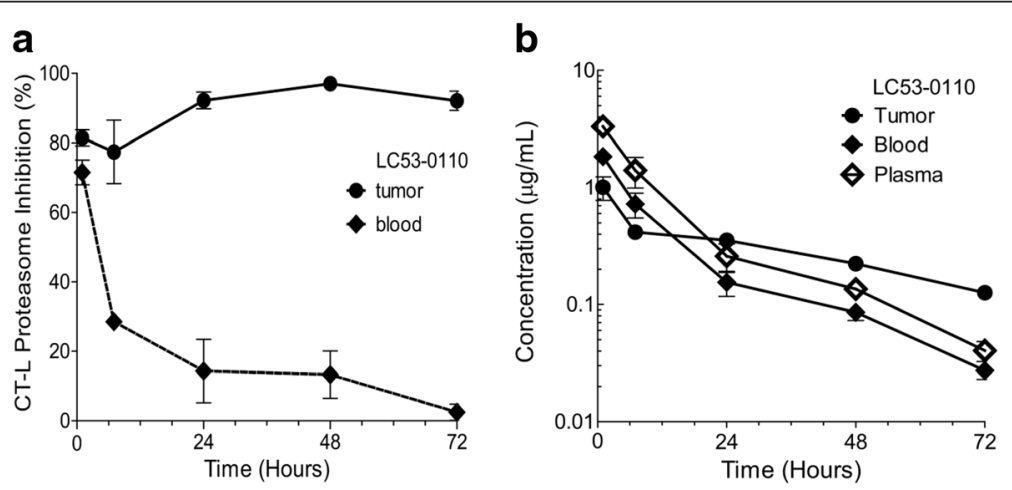

Fig. 4 PD and PK profiles of LC53-0110 in mice bearing human multiple myeloma xenografts. a MM.1S tumor bearing mice were administered orally with a single dose of LC53-0110 at $50 \mathrm{mg} / \mathrm{kg}$, and the 20S proteasome catalytic activities in tumor and blood were evaluated with CT-L subunit-specific fluorogenic peptide substrates. Data points, mean +/-SE $(n=3)$. $\mathbf{b}$ In the same study, concentration-time profiles of LC53-0110 in plasma, blood, and tumor were determined. Data points, mean $+/-S D(n=3)$ 


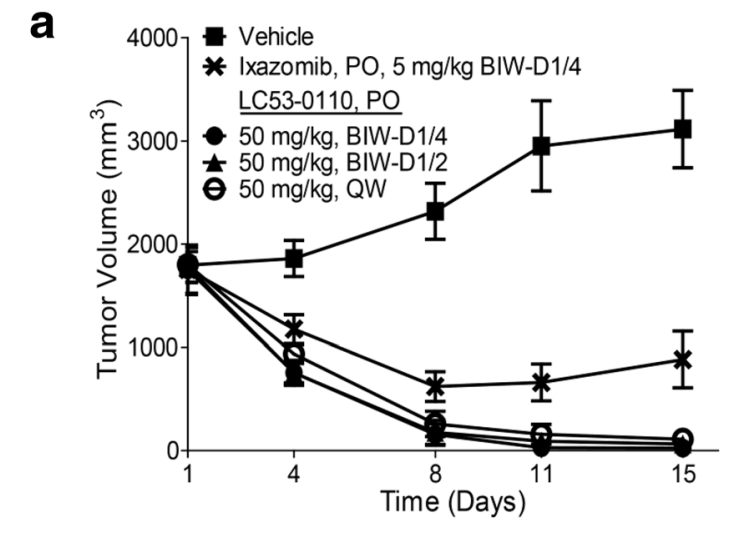

b

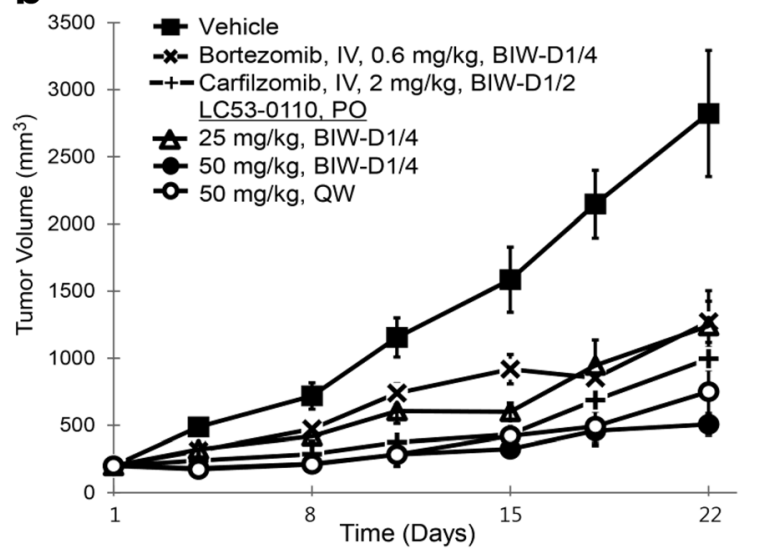

Fig. 5 Anti-tumor activity of LC53-0110 in mice bearing human multiple myeloma xenografts. a The LC and reference compounds were orally administered twice a week (BIW) or once a week (QW) in mice bearing MM.1S xenografts as indicated. The mean tumor volume for each animal in the vehicle and treatment groups was determined during the study. Data points, mean+/-SE $(n=6)$. b LC53-0110 was orally administered twice a week or once a week in mice bearing RPMI8226 xenografts as indicated, and reference compounds were intravenously administered twice a week. The mean tumor volume for each animal in the vehicle and treatment groups was determined during the study. Data points, mean+/-SE $(n=8)$

\section{An analog of LC53-0110 synergizes with an} immunomodulatory drug for anti-tumor activity

To address if an LC compound enhances anti-tumor activity when combined with immunomodulatory drugs, MM.1S cells were treated with pomalidomide, a thirdgeneration immunomodulatory drug, for $24 \mathrm{~h}$ and then with the LC53-0110 analog LC53-0151 for an additional $24 \mathrm{~h}$ across a range of concentrations. When cell proliferation was measured by MTT analysis, cells treated with LC53-0151 or pomalidomide as a single agent showed lower viability as compared to DMSO-treated control cells, and the combination of both agents showed a greater inhibition of viability than either agent alone (Fig. 6a). Moreover, isobologram analysis confirmed that LC53-0151 and pomalidomide induced synergistic cytotoxicity in MM.1S cells (combination index analyzed by CalcuSyn $\mathrm{S} / \mathrm{W}<1.0$ ).
In order to address if the observed synergism is also displayed in vivo, the efficacy of oral repeat-dose LC530151 was evaluated with regard to tumor growth inhibition in MM.1S xenografted nude mice with or without pomalidomide treatment. LC53-0151 and pomalidomide were orally administered for 3 weeks at $20 \mathrm{mg} / \mathrm{kg}$ twice a week and at $2.5 \mathrm{mg} / \mathrm{kg}$ for 5 days per week, respectively. Whereas pomalidomide treatment displayed $10 \%$ reduction of tumor volume with no statistically significance as compared to vehicle-treated mice, LC53-0151 treatment exhibited $43 \%$ reduction of tumor volume (Fig. 6b). In addition to its anti-tumor efficacy as a monotherapy, LC53-0151 potentiated the anti-tumor efficacy of pomalidomide, resulting in a $63 \%$ reduction of tumor volume on day 22 as compared to vehicle-treated mice. The improved efficacy of LC53-0151 when combined with pomalidomide was also confirmed by weight measurement of tumors extracted after final tumor volume measurement in tumor-bearing mice (Fig. 6c).

\section{Discussion}

Since bortezomib came on the market, there has been significant improvement in survival of MM patients [6]. However, the drug has some drawbacks such as toxicities including gastrointestinal symptoms (e.g., diarrhea), fatigue, thrombocytopenia, anemia, and peripheral neuropathy (PN), which is a common complication [22, 23]. In addition, it must be administered by intravenous or subcutaneous injection. Carfilzomib, another proteasome inhibitor on the market, has a low incidence of PN, but it has common serious adverse events (AEs) including fever, pneumonia, acute renal failure, and congestive heart failure, and it also must be administered by intravenous injection [24-27]. Since proteasome inhibitors emerged as an important component of MM treatment, logistical challenges for those injection approaches have become clear, especially for patients who need long-term therapy. Recent studies of the investigational oral proteasome inhibitor ixazomib, an improved version of bortezomib, showed a lower incidence of grade $3 \mathrm{PN}$ as compared to bortezomib, and drug-related other grade 3 AEs including diarrhea, fatigue, thrombocytopenia, neutropenia, and skin rash [28-33]. With improving survival in MM, the oral route of administration of ixazomib is considered to be high value, especially with the all-oral triple combination of ixazomib, an immunomodulatory agent, and a steroid being the most convenient and effective [34]. This is a particularly important factor for post-transplant maintenance, which can last several years. Therefore, we focused on developing an oral proteasome inhibitor that can be differentiated from ixazomib on the efficacy and safety profiles.

LC53-0110 is a more specific inhibitor of CT-L as compared to ixazomib, which has a similar potency toward C-L as CT-L (Table 1). The biological role of the 

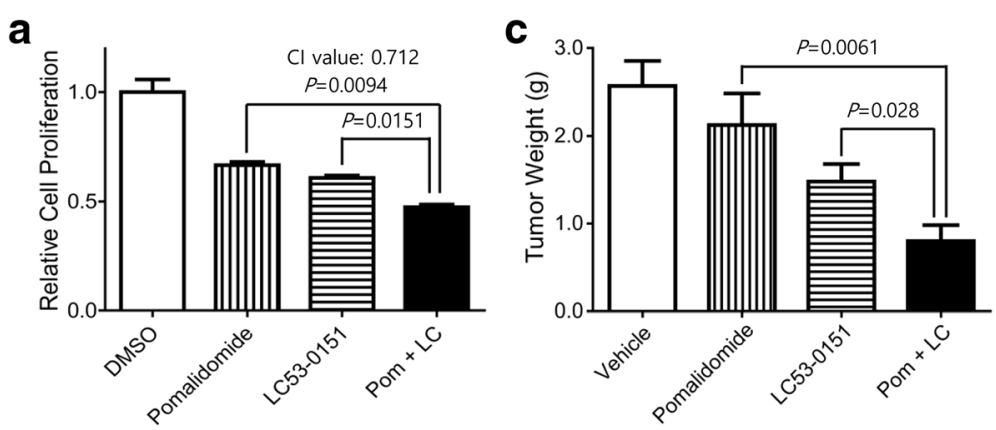

b

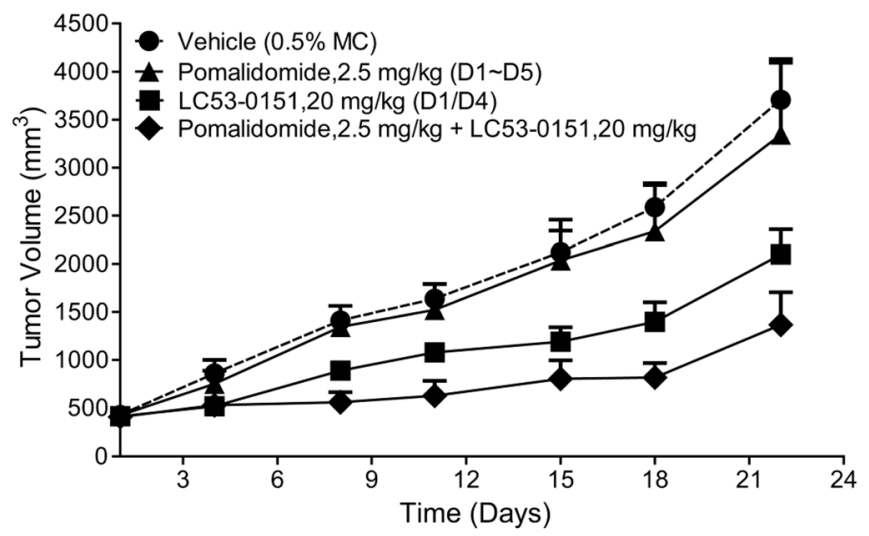

Fig. 6 Anti-tumor activity of an LC53-0110 analog combined with an immunomodulatory drug. a The cytotoxic effects of 5 nM of LC53-0151 or $2 \mu \mathrm{M}$ of pomalidomide alone or together on MM.1S cells were determined by MTT assay. $\mathbf{b}$ LC53-0151 or pomalidomide alone or together in mice bearing MM.1S xenografts as indicated. The mean tumor volume for each animal in the vehicle and treatment groups was determined during the study. $\mathbf{c}$ The mean tumor weight for each animal in the vehicle and treatment groups was determined at the end of the study. Data points, mean+/-SE $(n=8)$

individual proteasomal active sites is not fully understood. No phenotypic or proteolytic defects were observed upon inactivation of $\mathrm{C}$-L sites by site-directed mutagenesis of catalytic threonines in the yeast S. cerevisiae [35, 36]. Using chemical inhibitors or activity-based probes of C-L site, there have been some efforts to address the question of whether inhibition of this site is important for antitumor activity, especially in conjunction with inhibition of CT-L site [37-40]. However, due to the properties of the selective compounds including potency, cell permeability, stability, ADME/PK, and toxicity, the physiological consequences of C-L inhibition still need to be established. The study with carfilzomib, a relatively specific CT-L inhibitor like LC53-0110, supported the notion that inhibition of cellular CT-L activity is sufficient to kill hematological tumor cells, in particular MM cells, with minimal effects on normal leukocytes [41]. This is consistent with the findings in our study with LC53-0110, i.e., accumulation of ubiquitinated proteins, decrease of cell viability, and increase of apoptosis in MM cells (Table 2, Fig. 2).

LC53-0110 with CT-L specificity also showed potent inhibition of viability of MM cells obtained from patients in a dose-dependent manner (Fig. 3). Although it is risky to make a conclusion with a limited number of patients, patients who were newly diagnosed (patients 11 and 4) showed the lower end of cellular potency (i.e., most potent) as compared to those patients with multiple prior therapies (2-6 regimens) including proteasome inhibitors, thalidomide, lenalidomide, dexamethasone, histone deacetylase inhibitor, and chemotherapeutic drugs. Interestingly, LC53-0110 showed increased potency in MM cells obtained from bortezomib- and ixazomib-refractory patient with $10 \%$ viability at $12.5 \mathrm{nM}$, at which ixazomib did not show any effect on viability (patient 14, Fig. 3a). LC53-0110 clearly showed the ability to inhibit viability of MM cells of patients who were progressed after various treatments, with potency similar to those of MM cell lines. Therefore, it is likely that the proteasome CT-L active sites inhibited by LC53-0110 were not affected in these patients.

In human MM xenograft models in nude mice, oral administration of LC53-0110 showed much greater proteasome inhibition in tumor compared with blood, which was also the case for ixazomib [42] (Fig. 4a; and data not shown). However, LC53-0110 showed greater maximum and sustained tumor proteasome inhibition as compared with ixazomib ( $>90 \%$ with a duration of action of at least $72 \mathrm{~h}$ ). On repeat dosing, LC53-0110 showed complete tumor growth regression in MM.1S xenografts, a greater effect as compared with ixazomib (Fig. 5a). The 
efficacious activity of LC53-0110 was also demonstrated by the lack of measurable tumor mass even six weeks after the final dose in the surviving mice (data not shown). LC53-0110 also showed greater tumor growth inhibition than bortezomib and carfilzomib in RPMI8226 xenografts at well-tolerated doses (Fig. 5b). However, in this efficacy model, all 8 mice died or were euthanized due to moribund condition the day after the first oral treatment with ixazomib at $5 \mathrm{mg} / \mathrm{kg}$, which most likely is related to the possible toxicity of ixazomib.

Various agents have been proven to enhance antitumor activity in combination with other agents, and the addition of immunomodulatory drugs such as thalidomide or lenalidomide is considered one of the standard MM therapies [43-48]. Recently, treatment of relapsed MM with the proteasome inhibitor carfilzomib in combination with lenalidomide and dexamethasone showed better progression-free survival and overall response than lenalidomide and dexamethasone alone [49]. Pomalidomide is a new generation of immunomodulatory drug which was shown to confer a survival advantage even in patients who are refractory to both bortezomib and lenalidomide [50]. Pomalidomide is currently being investigated in combination with dexamethasone [51, 52], with bortezomib and dexamethasone [53], or with carfilzomib and dexamethasone [54] in patients with relapsed/refractory MM. LC53-0151, an analog of LC53-0110, in combination with pomalidomide also showed further synergistic inhibition of tumor growth both in vitro and in the xenograft mouse model (Fig. 6).

\section{Conclusions}

The orally available LC53-0110 and its analog LC530151 having strong efficacy and toxicity profiles may further improve the therapeutic efficacy, safety, and convenience for patients when used in combination with orally available immunomodulatory drugs. Therefore, in view of the in vitro, in vivo, and ex vivo profiles, further investigation of additional LC compounds in preclinical studies is warranted for the nomination of a clinical development candidate.

\section{Additional files}

Additional file 1: Figure S1. Activation of apoptosis in cells. Lysates were prepared from RPMI8226 cells $24 \mathrm{~h}$ after a 1-h treatment with $0.1 \%$ DMSO or $500 \mathrm{nM}$ of proteasome inhibitors. Western blot analysis was performed with anti-caspase-8 and anti-caspase-9 antibodies (A), or anti-PARP antibody (B). aTubulin was used as a loading control. Fl: full-length, Cl: cleaved. (PDF 31 kb)

Additional file 2: Figure S2. Inhibition of cellular viability in patientderived samples. CD138 ${ }^{+}$cells were purified from the bone marrow samples of MM patients $(3,4,6-8,11,14-16)$. The cytotoxic effects of LC53-0110 were determined by bioluminescent measurement of cellular ATP using CellTiter-Glo reagent after $48 \mathrm{~h}$ of treatment. Results are expressed as \% cell viability over DMSO control. Cell viability was plotted for all LC53-0110 treated multiple myeloma patients. Data points, mean+/-SE. (PDF $41 \mathrm{~kb}$ )

\section{Abbreviations}

C-L: caspase-like; CT-L: chymotrypsin-like; MM: multiple myeloma; T-L: trypsin-like.

\section{Competing interests}

Employment-LG Life Sciences, Ltd. (BGK, C-KJ, EP, JP, K-AW, S-WK, THK, YJ); no competing interests to declare (C-KM, J-YL, S-EL)

\section{Authors' contributions}

BGK, C-KJ and S-WK synthesized the compounds. JP, EP, THK and YJ designed and performed experiments and analyzed the data. C-KM, S-EL, J-YL provided clinical practice and isolated primary CD138 cells. K-AW analyzed the data and wrote and revised the manuscript. All authors read and approved the final manuscript.

\section{Authors' information}

C-KM: Member of the Korean Society of Hematology, the Korean Association of Internal Medicine, Hematopoietic Stem Cell Transplantation Society for Transplantation Registration Committee, and MM Blood Institute Working Party Steering Committee.

K-AW: Member of American Association for Cancer Research, American Society of Clinical Oncology, and American Society of Hematology.

\section{Acknowledgements}

The authors would like to thank Seoul St. Mary's Hospital patients who have participated in the study.

\section{Author details}

${ }^{1}$ R\&D Center, LG Life Sciences, Ltd, Daejeon, South Korea. ²Department of Internal Medicine, Seoul St. Mary's Hospital, The Catholic University of Korea, Seoul, South Korea.

Received: 15 September 2015 Accepted: 17 March 2016

Published online: 24 March 2016

\section{References}

1. Ciechanover A. The ubiquitin-proteasome pathway: on protein death and cell life. EMBO J. 1998;17:7151-60.

2. Ciechanover A, Schwartz AL. The ubiquitin-proteasome pathway: the complexity and myriad functions of proteins death. Proc Natl Acad Sci U S A. 1998;95:2727-30.

3. Kisselev AF, Akopian TN, Castillo V, Goldberg AL. Proteasome active sites allosterically regulate each other, suggesting a cyclical bite-chew mechanism for protein breakdown. Mol Cell. 1999:4(3):395-402.

4. Teicher BA, Tomaszewski JE. Proteasome inhibitors. Biochem Pharmacol. 2015;96(1):1-9.

5. Adams J. The development of proteasome inhibitors as anticancer drugs. Cancer Cell. 2004;5(5):417-21.

6. Teicher BA, Anderson KC. CCR 20th anniversary commentary: In the beginning, there was PS-341. Clin Cancer Res. 2015;21(5):939-41.

7. Moreau $\mathrm{P}$, Richardson $\mathrm{PG}$, Cavo M, et al. Proteasome inhibitors in multiple myeloma: 10 years later. Blood. 2012;120:947-59.

8. Allegra A, Alonci A, Gerace D, et al. New orally proteasome inhibitors in multiple myeloma. Leuk Res. 2013;38:1-9.

9. Andreu-Vieyra C, Berenson JR. Carfilzomib in multiple myeloma. Expert Opin Biol Ther. 2014:14(11):1685-99.

10. Moreau P. The emerging role of carfilzomib combination therapy in the management of multiple myeloma. Expert Rev Hematol. 2014:7:265-90.

11. Sugumar D, Keller J, Vij R. Targeted treatments for multiple myeloma: specific role of carfilzomib. Pharmgenomics Pers Med. 2015:8:23-33.

12. Richardson PG, Moreau P, Laubach JP, et al. The investigational proteasome inhibitor ixazomib for the treatment of multiple myeloma. Future Oncol. 2015;11(8):1153-68.

13. Vij R, Savona M, Siegel DS, et al. Clinical profile of single-agent oprozomib in patients (pts) with multiple myeloma (MM): updated results from a multicenter, open-label, dose escalation phase $1 \mathrm{~b} / 2$ study [abstract]. Blood. 2014:124:Abstract 34

14. Millward M, Price T, Townsend A, et al. Phase 1 clinical trial of the novel proteasome inhibitor marizomib with the histone deacetylase inhibitor vorinostat in patients with melanoma, pancreatic and lung cancer based on in vitro assessments of the combination. Invest New Drugs. 2012;30(6): 2303-17. 
15. Sano R, Reed JC. ER stress-induced cell death mechanisms. Biochim Biophys Acta. 2013;1833(12):3460-70.

16. Mymrikov EV, Seit-Nebi AS, Gusev NB. Large potentials of small heat shock proteins. Physiol Rev. 2011;91(4):1123-59.

17. Zhang L, Fok JH, Davies FE. Heat shock proteins in multiple myeloma. Oncotarget. 2014;5(5):1132-48.

18. Stokoe D, Engel K, Campbell DG, Cohen P, Gaestel M. Identification of MAPKAP kinase 2 as a major enzyme responsible for the phosphorylation of the small mammalian heat shock proteins. FEBS Lett. 1992;313:307-13.

19. Rogalla T, Ehrnsperger M, Preville X, et al. Regulation of Hsp27 oligomerization, chaperone function, and protective activity against oxidative stress/tumor necrosis factor a by phosphorylation. J Biol Chem. 1999;274:18947-56.

20. Ashkenazi A. Targeting the extrinsic apoptotic pathway in cancer: lessons learned and future directions. J Clin Invest. 2015;125(2):487-9.

21. Fulda S, Vucic D. Targeting IAP proteins for therapeutic intervention in cancer. Nat Rev Drug Discov. 2012;11:109-24.

22. Kane RC, Bross PF, Farrell AT, Pazdur R. Velcade: U.S. FDA approval for the treatment of multiple myeloma progressing on prior therapy. Oncologist. 2003;8(6):508-13.

23. Kane RC, Farrell AT, Sridhara R, Pazdur R. United States Food and Drug Administration approval summary: bortezomib for the treatment of progressive multiple myeloma after one prior therapy. Clin Cancer Res. 2006:12(10):2955-60.

24. Herndon TM, Deisseroth A, Kaminskas E, et al. U.S. Food and Drug Administration approval: carfilzomib for the treatment of multiple myeloma. Clin Cancer Res. 2013;19(17):4559-63.

25. Nooka A, Gleason C, Casbourne D, Lonial S. Relapsed and refractory lymphoid neoplasms and multiple myeloma with a focus on carfilzomib. Biologics. 2013;7:13-32.

26. Siegel $D$, Martin T, Nooka A, et al. Integrated safety profile of single-agent carfilzomib: experience from 526 patients enrolled in 4 phase II clinical studies. Haematologica. 2013;98(11):1753-61.

27. Atrash S, Tullos A, Panozzo S, et al. Cardiac complications in relapsed and refractory multiple myeloma patients treated with carfilzomib. Blood Cancer J. 2015;5:e272.

28. Offidani M, Corvatta L, Caraffa P, Gentili S, Maracci L, Leoni P. An evidencebased review of ixazomib citrate and its potential in the treatment of newly diagnosed multiple myeloma. Onco Targets Ther. 2014;7:1793-800.

29. Richardson $P G$, Baz R, Wang M, et al. Phase 1 study of twice-weekly ixazomib, an oral proteasome inhibitor, in relapsed/refractory multiple myeloma patients. Blood. 2014;124(7):1038-46.

30. Kumar SK, Bensinger WI, Zimmerman TM, et al. Phase 1 study of weekly dosing with the investigational oral proteasome inhibitor ixazomib in relapsed/refractory multiple myeloma. Blood. 2014;124(7):1047-55.

31. Kumar SK, Berdeja JG, Niesvizky R, et al. Safety and tolerability of ixazomib, an oral proteasome inhibitor, in combination with lenalidomide and dexamethasone in patients with previously untreated multiple myeloma: an open-label phase 1/2 study. Lancet Oncol. 2014;15(13):1503-12.

32. Kumar SK, Berdeja JG, Niesvizky R, et al. Long-term ixazomib maintenance is tolerable and improves depth of response following ixazomib-lenalidomidedexamethasone induction in patients (Pts) with previously untreated multiple myeloma (MM): phase 2 study results [abstract]. Blood. 2014;124:Abstract 82.

33. Handa H, Suzuki K, Chou T, Matsushima T. Phase 1 study of the investigational proteasome inhibitor ixazomib alone or in combination with lenalidomidedexamethasone (Rd) in Japanese patients (Pts) with relapsed and/or refractory multiple myeloma (RRMM) [abstract]. Blood. 2014;124:Abstract 5752.

34. Moreau P. Oral therapy for multiple myeloma: ixazomib arriving soon [commentary]. Blood. 2014;124(7):986-7.

35. Arendt CS, Hochstrasser M. Identification of the yeast $20 \mathrm{~S}$ proteasome catalytic centers and subunit interactions required for active-site formation. Proc Natl Acad Sci U S A. 1997;94(14):7156-61.

36. Heinemeyer W, Fischer M, Krimmer T, Stachon U, Wolf DH. The active sites of the eukaryotic $20 \mathrm{~S}$ proteasome and their involvement in subunit precursor processing. J Biol Chem. 1997;272(40):25200-9.

37. van Swieten PF, Samuel E, Hernández RO, et al. A cell-permeable inhibitor and activity-based probe for the caspase-like activity of the proteasome. Bioorg Med Chem Lett. 2007;17(12):3402-5.

38. Britton M, Lucas MM, Downey SL, et al. Selective inhibitor of Proteasome's caspase-like sites sensitizes cells to specific inhibition of chymotrypsin-like sites. Chem Biol. 2009:16(12):1278-89.
39. Baldisserotto A, Ferretti V, Destro F, et al. Alpha, beta-unsaturated Nacylpyrrole peptidyl derivatives: new proteasome inhibitors. J Med Chem. 2010;53(17):6511-5.

40. van der Linden WA, Willems $\mathrm{LI}$, Shabaneh TB, et al. Discovery of a potent and highly $\beta 1$ specific proteasome inhibitor from a focused library of ureacontaining peptide vinyl sulfones and peptide epoxyketones. Org Biomol Chem. 2012;10(1):181-94.

41. Parlati F, Lee SJ, Aujay M, et al. Carfilzomib can induce tumor cell death through selective inhibition of the chymotrypsin-like activity of the proteasome. Blood. 2009; 114(16):3439-47.

42. Kupperman $\mathrm{E}$, Lee EC, Cao Y, et al. Evaluation of the proteasome inhibitor MLN9708 in preclinical models of human cancer. Cancer Res. 2010;70(5):1970-80

43. Stewart AK, Richardson PG, San-Miguel JF. How I treat multiple myeloma in younger patients. Blood. 2009;114(27):5436-43.

44. Andhavarapu S, Roy V. Immunomodulatory drugs in multiple myeloma. Expert Rev Hematol. 2013;6(1):69-82.

45. Cavo M, Tacchetti P, Patriarca F, et al. Bortezomib with thalidomide plus dexamethasone compared with thalidomide plus dexamethasone as induction therapy before, and consolidation therapy after, double autologous stem-cell transplantation in newly diagnosed multiple myeloma: a randomised phase 3 study. Lancet. 2010;376:2075-85.

46. Rosinol L, Oriol A, Teruel Al, et al. Superiority of bortezomib, thalidomide, and dexamethasone (VTD) as induction pretransplantation therapy in multiple myeloma: a randomized phase 3 PETHEMA/GEM study. Blood. 2012;120:1589-96

47. Richardson $\mathrm{PG}$, Weller $\mathrm{E}$, Lonial $\mathrm{S}$, et al. Lenalidomide, bortezomib, and dexamethasone combination therapy in patients with newly diagnosed multiple myeloma. Blood. 2010;116:679-86.

48. Kumar S, Flinn I, Richardson PG, et al. Randomized, multicenter, phase 2 study (EVOLUTION) of combinations of bortezomib, dexamethasone, cyclophosphamide, and lenalidomide in previously untreated multiple myeloma. Blood. 2012;119:4375-82.

49. Stewart AK, Rajkumar SV, Dimopoulos MA, et al. Carfilzomib, lenalidomide, and dexamethasone for relapsed multiple myeloma. N Engl J Med. 2015; 372:142-52.

50. Fouquet $\mathrm{G}$, Bories $\mathrm{C}$, Guidez $\mathrm{S}$, et al. Pomalidomide for multiple myeloma. Expert Rev Hematol. 2014;7(6):719-31.

51. San Miguel J, Weisel K, Moreau P, et al. Pomalidomide plus low-dose dexamethasone versus high-dose dexamethasone alone for patients with relapsed and refractory multiple myeloma (MM-003): a randomised, openlabel, phase 3 trial. Lancet Oncol. 2013;14(11):1055-66.

52. Weisel K, Moreau P, Gibson CJ, et al. Analyzing the relationship of response and survival in patients with refractory or relapsed and refractory multiple myeloma (RRMM) treated with pomalidomide plus low-dose dexamethasone (POM + LoDEX) in the MM-003 trial [abstract]. J Clin Oncol. 2015;33:Abstract 8593.

53. Richardson PG, Bensmaine A, Doerr T, Wang J, Zaki MH. MM-007: A phase 3 trial comparing the efficacy and safety of pomalidomide (POM), bortezomib (BORT), and low-dose dexamethasone (LoDEX [PVD]) versus BORT and LoDEX (VD) in subjects with relapsed or refractory multiple myeloma (RRMM) [abstract]. J Clin Oncol. 2015;33:Abstract TPS8610.

54. Stewart AK. Carfilzomib for the treatment of patients with relapsed and/or refractory multiple myeloma. Future Oncol. Prepublished on June 30, 2015, as doi:10.2217/fon. 15.123

\section{Submit your next manuscript to BioMed Central and we will help you at every step:}

- We accept pre-submission inquiries

- Our selector tool helps you to find the most relevant journal

- We provide round the clock customer support

- Convenient online submission

- Thorough peer review

- Inclusion in PubMed and all major indexing services

- Maximum visibility for your research

Submit your manuscript at www.biomedcentral.com/submit 